\title{
Sharp and seismically transparent inner core boundary region revealed by an entire network observation of near-vertical PKiKP
}

\author{
Hitoshi Kawakatsu \\ Earthquake Research Institute, University of Tokyo, 1-1-1 Yayoi, Bunkyo-ku, Tokyo 113-0032, Japan \\ (Received August 12, 2005; Revised February 6, 2006; Accepted February 15, 2006; Online published July 26, 2006)
}

\begin{abstract}
We present an entire network observation of near-vertical PKiKP from the Japanese seismic network, Hinet. The record section of an intermediate depth $(298 \mathrm{~km})$ earthquake in Mariana (2001/07/03 13:10:43, Mw 6.5) shows remarkably clear arrivals of PKiKP in an epicentral distance range of 14-24 degrees. From more than 170 individual picks of PKiKP, we performed array analyses of PKiKP-related seismic phases to infer the structure near the inner core boundary (ICB) with unprecedented high quality. Both PKiKP and PcP showed strong similarity in waveforms up to a frequency of $2 \mathrm{~Hz}$. Both amplitude and waveform analyses indicate that the ICB may be as sharp as the CMB and no thicker than $2 \mathrm{~km}$. The slant stack for a $1-2 \mathrm{~Hz}$ frequency band, where PKiKP and PcP show energy peaks and where the background noise level is $\sim 3 \%$ of PKiKP, indicated no conspicuous phase other than SKiKP. We also noted that no gradual energy build-up of the inner core scattering signal was observed. Thus, this part of the inner core appears highly transparent seismically in this frequency range. In the $0.5-1.0 \mathrm{~Hz}$ frequency band (10-15\% noise level), we observed one possible reflection phase from a slightly dipping $\left(\sim 5^{\circ}\right)$ reflector/discontinuity inside of the inner core around a depth of $470 \mathrm{~km}$ below the ICB (3\% reflection). However, this phase may be due to pPKiKP water/crustal layer reverberations. Other than this, there appears to be no sharp ( $\sim 5 \mathrm{~km}$ thick) horizontal discontinuity in the top $400 \mathrm{~km}$ part of the inner core whose reflection amplitude is larger than $\sim 2 \%$.
\end{abstract}

Key words: Inner Core, PKiKP, ICB, stacking, array analysis.

\section{Introduction}

The inner core boundary (ICB), which separates the solid inner core from the fluid outer core, is the least wellunderstood major boundary of the solid Earth. It is generally accepted that the inner core has been growing through the history of the Earth as it cools down, and as a result, the ICB surface has been renewed continually at least over the last billion years (e.g., Labrosse and Macouin, 2003). Based on well-known results in metallurgy, Fearn et al. (1981) suggested that the condition at the ICB is likely to satisfy that for a morophological instability of a planar interface between a molten alloy and the corresponding solid to develop a so-called "mushy zone" in which both solid and liquid phase coexist. Recently, Shimizu et al. (2005) conducted a stability analysis to confirm the existence of a mushy zone at the ICB. The theoretically estimated thickness of this mushy zone varies from $\sim 1000 \mathrm{~km}$ (Fearn et al., 1981) to $\sim 10 \mathrm{~m}$ (Sumita et al., 1996), indicating the necessity for other information to constrain the actual conditions at the ICB.

Since the discovery of the solid inner core by Lehman (1936), the seismic P-wave velocity structure of the inner core has been mostly constrained by the observations of "core phases" recorded at remote seismic stations from sources $\left(\Delta>\sim 110^{\circ}\right)$. These core phases either travel

Copyright (c) The Society of Geomagnetism and Earth, Planetary and Space Sciences (SGEPSS); The Seismological Society of Japan; The Volcanological Society of Japan; The Geodetic Society of Japan; The Japanese Society for Planetary Sciences; TERRAPUB. through the inner core as a P-wave (called PKIKP or PKPdf), or are (post-critically) reflected at the ICB (PKiKP or PKP-cd). Studies of these phases not only constrained the spherically averaged P-wave structure of the inner core (e.g., PREM, AK135), but also revealed non-spherical structures of the inner core, such as anisotropic structure (e.g., Morelli et al., 1986; Creager, 1992; Song and Helmberger, 1993), the possible differential rotation (e.g., Song and Richards, 1996), and hemispherical lateral heterogeneity (e.g., Tanaka and Hamaguchi, 1997; Niu and Wen 2001).

On the other hand, to constrain the seismic structure near the ICB, the most useful phase is the P-wave reflected from the top of the inner core recorded at stations not too remote from earthquakes $\left(\Delta<\sim 70^{\circ}\right)$. Observation of this phase, (pre-critical) PKiKP, enables us to constrain the radius of the inner core, as well as the density jump at the ICB which is a crucial parameter to investigate the energy source of the geodynamo. Clear observation of this phase is, however, known for its rareness due to its small amplitude, preventing detailed seismological investigation of the top part of the inner core. By using the LASA seismic array, Engdahl et al. (1970) reported clear observations of the near-vertical PKiKP, and confirmed the previously estimated radius of the inner core. They also noted that the ICB must be sharp enough to reflect seismic energy at $\sim 1 \mathrm{~Hz}$ (i.e., the ICB thickness $<5 \mathrm{~km}$; Cummins and Johnson, 1988). Since their work, there have been attempts (not too many) to further constrain the physical properties of the ICB (e.g., Souriau and Souriau, 1989; Shearer and Masters, 1990). 
Analyzing array data from the International Monitoring System (IMS), Koper and his colleagues recently increased the number of PKiKP observations by an order of magnitude (Koper et al., 2003; Koper and Pyle, 2004; Koper and Dombrovskaya, 2005). Based on the large number of PKiKP and PcP observations, they were able to constrain the aspherical structure of the core, and also constructed a seismic model for the ICB. Another interesting issue regarding PKiKP and the ICB region is the observation of scattered energy first reported by Vidale and Earle (2000), who suggested the presence of scatterers in the top $300 \mathrm{~km}$ of the inner core. Recently, Poupinet and Kennett (2004) reported high-frequency $(1-5 \mathrm{~Hz})$ coda of PKiKP at short distances $\left(<45^{\circ}\right)$, and attributed it to complex reverberation effects near the ICB (see also Koper et al., 2004).

Here we present an unusual entire network observation of such near-vertical PKiKP, first reported by Kawakatsu and Watada (2002). The seismic section of an intermediate depth $(298 \mathrm{~km})$ earthquake in Mariana (2001/07/03 13:10:43, Mw 6.5) recorded by the then Japanese Hi-net (Obara et al., 2005) shows remarkably clear arrivals of PKiKP in an epicentral distance range of 14-24 degrees. From more than 170 individual picks of PKiKP, this data set allowed us not only to study the ICB in a similar way as the previous investigators but also to see the structure below the ICB with unprecedented high quality.

\section{Entire Network Observation of Near-vertical PKiKP}

Figure 1 shows the station distribution of Hi-net used in the present analysis. Hi-net consists of nearly 700 shortperiod seismometers (1 Hz, 3-component) buried in deep boreholes around a depth of $100 \mathrm{~m}$, covering the Japanese islands with a nominal spacing of $\sim 20 \mathrm{~km}$ (Obara et al., 2005). At the time of the earthquake, the deployment of the network was still underway, and records of less than 500 stations were available through the Internet.

Figure 2 is the record section of the vertical component seismograms of the recording of the Mariana earthquake. Although PcP is partly masked by the S-wave energy, clear arrivals of ScP and somewhat diffused energy of $\mathrm{ScS}$ may be recognized. The arrivals around $960 \mathrm{sec}$ from the origin time, which have a small slowness, indicating a nearvertical incidence to the entire network, are consistent with those of reflections from the ICB, i.e., PKiKP. The identification that these phases are indeed PKiKP can be further evidenced by the slant stack (Fig. 3) of 78 seismograms for which PcP can be picked as a reference phase. Considering the rareness of PKiKP observation as mentioned above, this observation is remarkable and deserves further investigation.

\section{Sharpness of the ICB}

Figure 4(a) compares PcP and PKiKP waveforms obtained by stacking records of 44 stations $\left(14^{\circ}<\Delta<16^{\circ}\right)$ for which both phases can be clearly read. The strong similarity of the two waveforms indicates that the structure of both CMB and ICB in the studied region is similarly simple in the frequency range of interest. Figure 4(b) shows that the strongest energy of the two phases can be observed

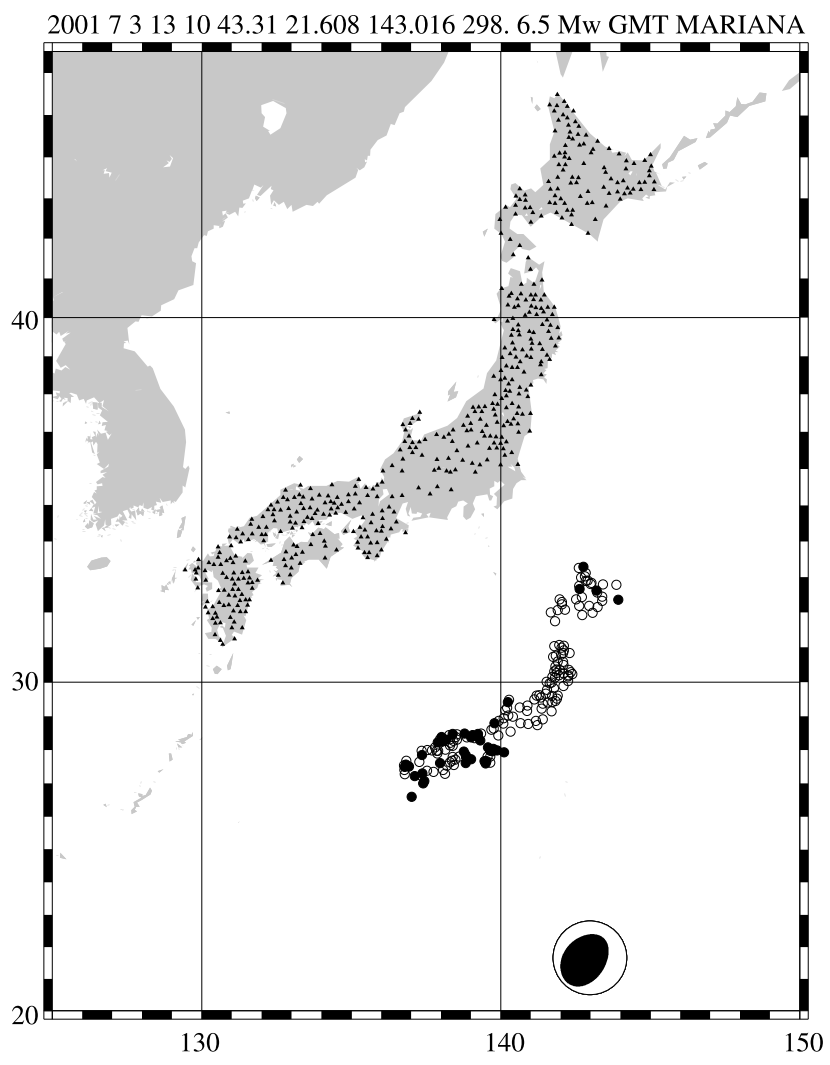

Fig. 1. A map of Hi-net stations (triangles) and PKiKP (open circles) and $\mathrm{PcP}$ (closed circles) bounce points. The PKiKP bounce points are of those 178 records for which PKiKP phases are actually picked for the analyses (there are more records that PKiKP are observed but not picked due to poor $\mathrm{S} / \mathrm{N}$ ratios). The $\mathrm{PcP}$ bounce points are of those stations for which both PcP and PKiKP are picked. The Harvard CMT solution of the Mariana earthquake is also plotted for the lower-hemisphere.

between 0.8 and $2.0 \mathrm{~Hz}$, indicating that the $\mathrm{CMB}$ and the ICB are relatively sharp $(\sim$ few $\mathrm{km})$. It is a common practice to use the amplitude ratio of $\mathrm{PKiKP} / \mathrm{PcP}$ to infer the density jump at the ICB (e.g., Souriau and Souriau, 1989). The observed ratio is 0.125 which is comparable within the error bounds to the value (0.118) obtained for DSM synthetic seismograms (Takeuchi et al., 1996) calculated for PREM (Dziewonski and Anderson, 1981). For comparison, the same station set (44 stations) were used to make stacked PKiKPs and PcPs of both observed and synthetic seismograms. Thus, the present data appear to be consistent with the PREM values at the ICB.

In order to further infer the sharpness of the ICB, Fig. 5 presents the effect of varying the ICB transition zone thickness on the PKiKP waveform and amplitude. We use the observed stacked PcP of Fig. 4(a) as an input and calculate simulated PKiKPs for different values of the transition thickness. We use the Haskell matrix for the simulation, and assume a linear gradient for the ICB transition zone. A $1 \mathrm{~km}$-thick ICB reduces the amplitude of the first phase by about $13 \%$, which is about the observation error bounds $(2 \sigma \sim 10 \%)$. Thus, if we accept the PREM ICB values, the observed ratio of $\mathrm{PKiKP} / \mathrm{PcP}$ suggests that the ICB is less than $1 \mathrm{~km}$ thick. As the CMB is known to have very complicated structures, and as the change of the CMB structure affects the ratio significantly for short distant ranges (Koper 


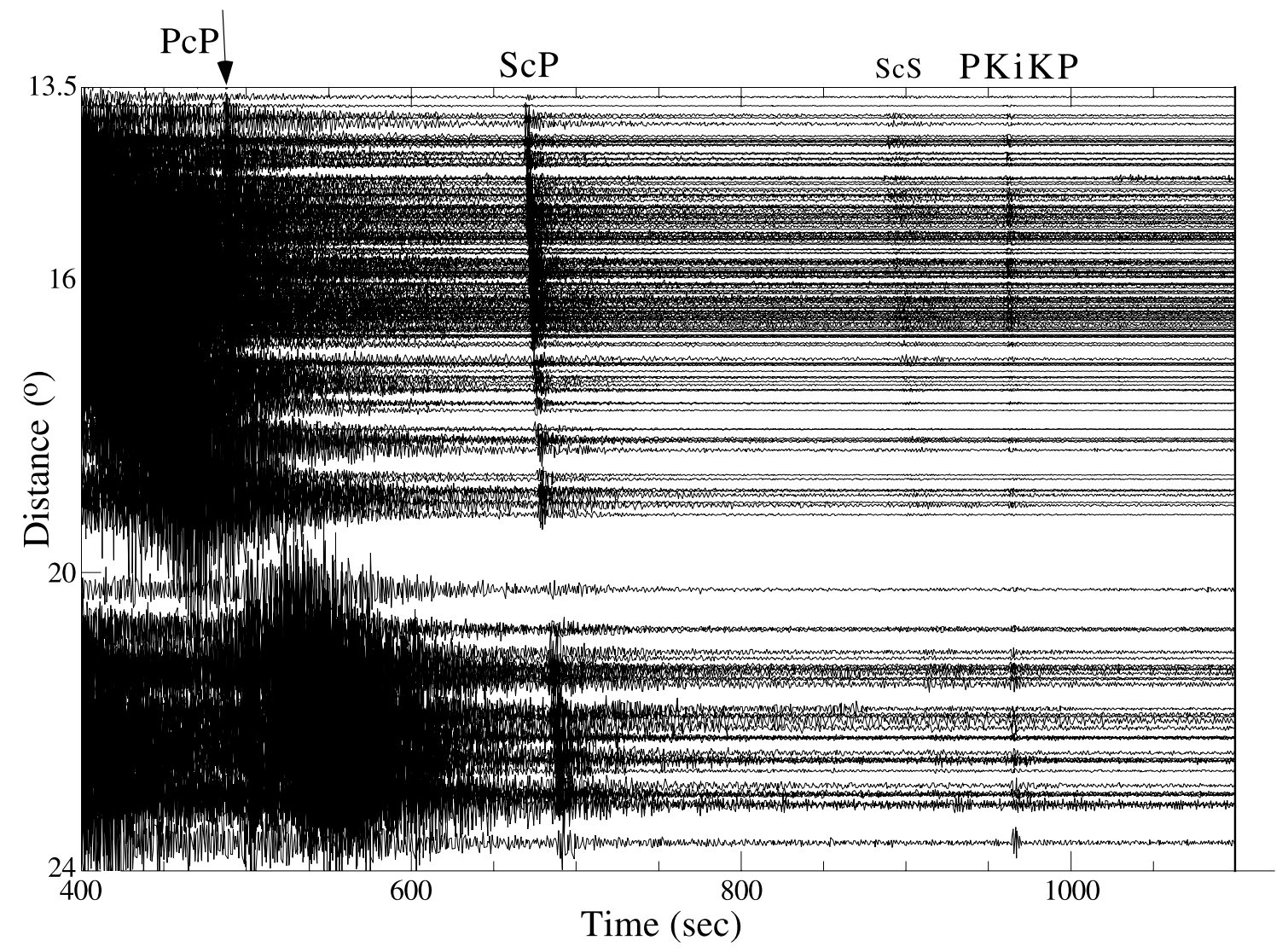

Fig. 2. Seismic section of the vertical component recordings of the Mariana event. Beyond $\sim 16^{\circ} \mathrm{PcP}$ are masked by the large S-wave arrivals. The clear arrivals about $960 \mathrm{~s}$ after the origin time are those of PKiKP.

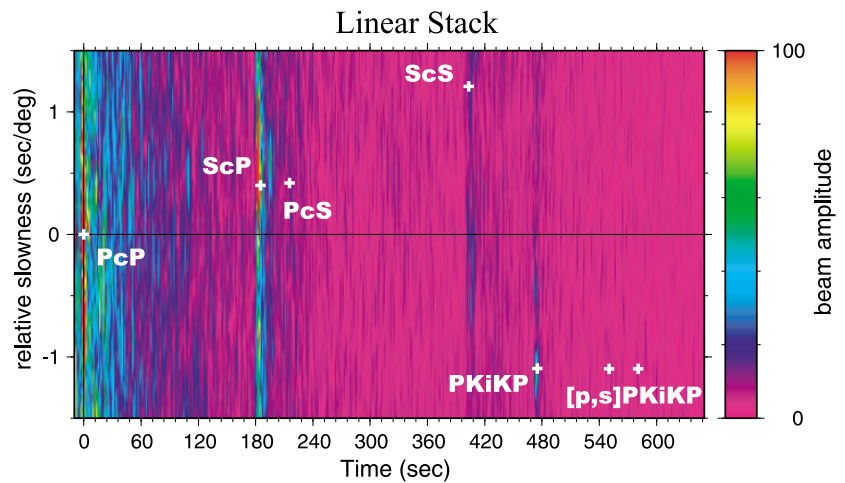

Fig. 3. Vespagram constructed from 88 seismograms for which PcP are readable. White plus marks denote location of major reflection phases. Note that PKiKP are clearly identified.

and Pyle, 2004), this effect should also be taken into account. On the other hand, the observed PcP and PKiKP waveforms and spectra do not show any indication of complicated $\mathrm{CMB}$ or ICB structures in the studied region. Further, PcP bounce points on the CMB (Fig. 1) do not seem to correspond to a very heterogeneous area (e.g., Obayashi and Fukao, 1997; Thorne and Garnero, 2004). We thus may estimate the upper bound of the CMB heterogeneity $\sim 1 \%$, which changes the reflection coefficient $\sim 8 \%$. Therefore, a $2 \mathrm{~km}$-thick ICB (amplitude reduction of $\sim 40 \%$ ) appears to be inconsistent with the observed $\mathrm{PKiKP} / \mathrm{PcP}$ ratio (assuming the PREM ICB values).
Waveform comparison The consistent two pulses seen in the PcP and PKiKP waveforms are most likely due to a complex source effect. The simulated PKiKP in Fig. 5(a) shows a slight difference in the amplitude of these two pulses for different transition thicknesses. This is due to the frequency dependence of the reflection coefficient for a transitional ICB and to the slight difference of the frequency content of the two pulses. Based on these observations, we directly compared waveforms of observed and simulated PKiKP to obtain further constraint on the ICB thickness. Close inspection of these two pulses, however, revealed that their relative timings are slightly shifted for PcP and PKiKP; the second pulse of PKiKP arrives a little earlier compared to that of PcP (Fig. 4(a)). This small shift $(0.15 \mathrm{~s})$ of the pulse between the two phases can be accounted for if the sources of the two pulses are spatially separated by about $15 \mathrm{~km}$. Yamanaka (2005, personal communication) modeled teleseismic waveforms of this event, and indeed obtained two sub-events spatially separated by about $15 \mathrm{~km}$ (http://www.eri.u-tokyo.ac.jp/sanchu/ 2Seismo_Note/2001/010703.html, Earthquake Research Institute, 2005).

Considering this, the two waveforms are compared after shifting the second pulse of the simulated PKiKPs. Figure 6(a) shows two examples of such a comparison for the best-fit models for a sharp ICB and a $3 \mathrm{~km}$-thick ICB. It can be seen that the fit for a $3 \mathrm{~km}$-thick ICB is degraded and the ICB is unlikely to be this thick. Figure 6(b) summarizes the result; a sharp ( $0 \mathrm{~km}$ thick) ICB gives the best fit, although it 
(a)

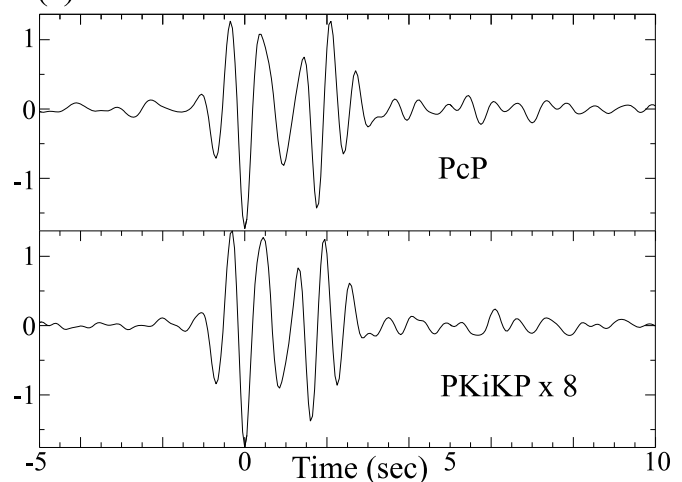

(b)

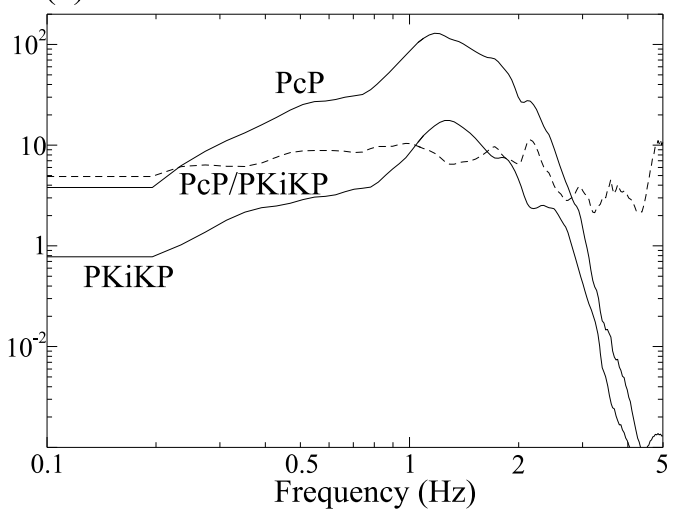

Fig. 4. Stacked velocity waveforms of PcP and PKiKP constructed from 44 seismograms for which both phases are readable. (a) Time domain waveforms (given in an arbitrary unit). The amplitude of PKiKP is multiplied by 8. (b) Amplitude spectra (given in an arbitrary unit). Note that the strongest energy are seen between 1-2 Hz.

is almost indistinguishable from the fit of a $1 \mathrm{~km}$-thick ICB. From this, we may conclude that the ICB may be as sharp as the CMB and is no thicker than $2 \mathrm{~km}$. This is consistent with the conclusion derived solely from the amplitude ratio argument.

\section{Reflection seismology of the inner core}

Figure 3 shows a linear stack vespagram obtained using $\mathrm{PcP}$ as a reference phase. As mentioned earlier, most previous work on pre-critical PKiKP take this approach to discuss the possible identification of a sharp boundary inside the inner core (e.g., Souriau and Souriau, 1989). In the present work, on the other hand, we can use PKiKP itself as a reference phase to investigate the structure below the ICB.

Figure 7(a) shows the result of a phase-weighted stack (Schimmel and Paulssen, 1997) using 78 PKiKP phase picks as a reference phase (the power index of 2 was used). The seismograms are band-pass filtered between 1 and $2 \mathrm{~Hz}$ where the seismic energy is the strongest (Fig. 4(b)). In the figure, crosses near the zero relative slowness line indicate possible locations of ICB related phases (PKiKP, $[p, s] P K i K P, S K i K P)$, and those below the line indicate locations of possible reflection phases from a horizontal reflector beneath the ICB (marks are shown in a $100 \mathrm{~km}$ depth interval). The strongest energy at about $180 \mathrm{~s}$ after PKiKP appears to correspond to SKiKP, and its rela-

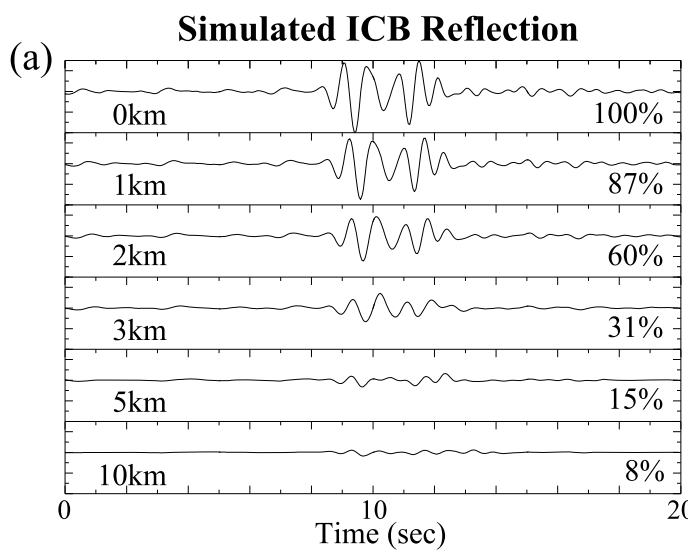

(b)

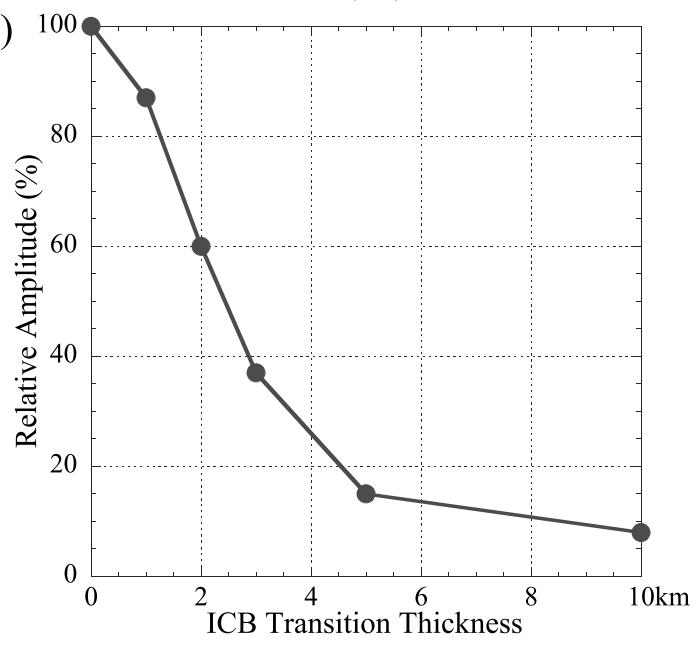

Fig. 5. The ICB transition thickness and PKiKP amplitude reduction. (a) Waveforms of simulated PKiKPs are shown for different ICB transition zone thicknesses. The stacked PcP waveform is used as the input. (b) The amplitude of the first pulse is given as a function of the ICB transition thickness.

tive amplitude to PKiKP is only about 4\% (Fig. 7(b)); this SKiKP/PKiKP amplitude ratio appears to be consistent with a known source mechanism (e.g., Harvard CMT) and a reference earth model (e.g., PREM). Other than this phase, no other notable energy may be identified (there is a slight indication of pPKiKP $\sim 75$ s). Figure 7(b) shows the waveform of a linear stack for the zero relative slowness; green lines indicate the amplitude of possible reflected phases from a sharp horizontal reflector with a $1 \%$ reflection (i.e., impedance contrast of $2 \%$; $1 \%$ both in velocity and density jumps if they scale together) at frequencies of 1 and $2 \mathrm{~Hz}(Q=360$ is assumed; Bhattacharyya et al., 1993). As the linear stack results for other slownesses show a similar feature, we may conclude that there is no sharp (few kilometers thick) discontinuity of the $1 \%$ reflection in the top $600 \mathrm{~km}$ (corresponding $\sim 100 \mathrm{~s}$ after PKiKP) of the inner core. Restricting to the top $400 \mathrm{~km}$, we may reduce a reflection down to $\sim 0.5 \%$.

The situation is a little different if we lower the frequency range of the analysis to $0.5-1.0 \mathrm{~Hz}$ (Fig. 8). Several peaks exist in the vespagram; among them those peaks at 75 and $85 \mathrm{~s}$ after PKiKP are most conspicuous (Fig. 8(a)). From the slowness and time, the one at $\sim 75 \mathrm{~s}$ is most likely to be pPKiKP. The phase observed $10 \mathrm{~s}$ later also shares a similar slowness, indicative of a similar origin (e.g., a crustal 
(a)
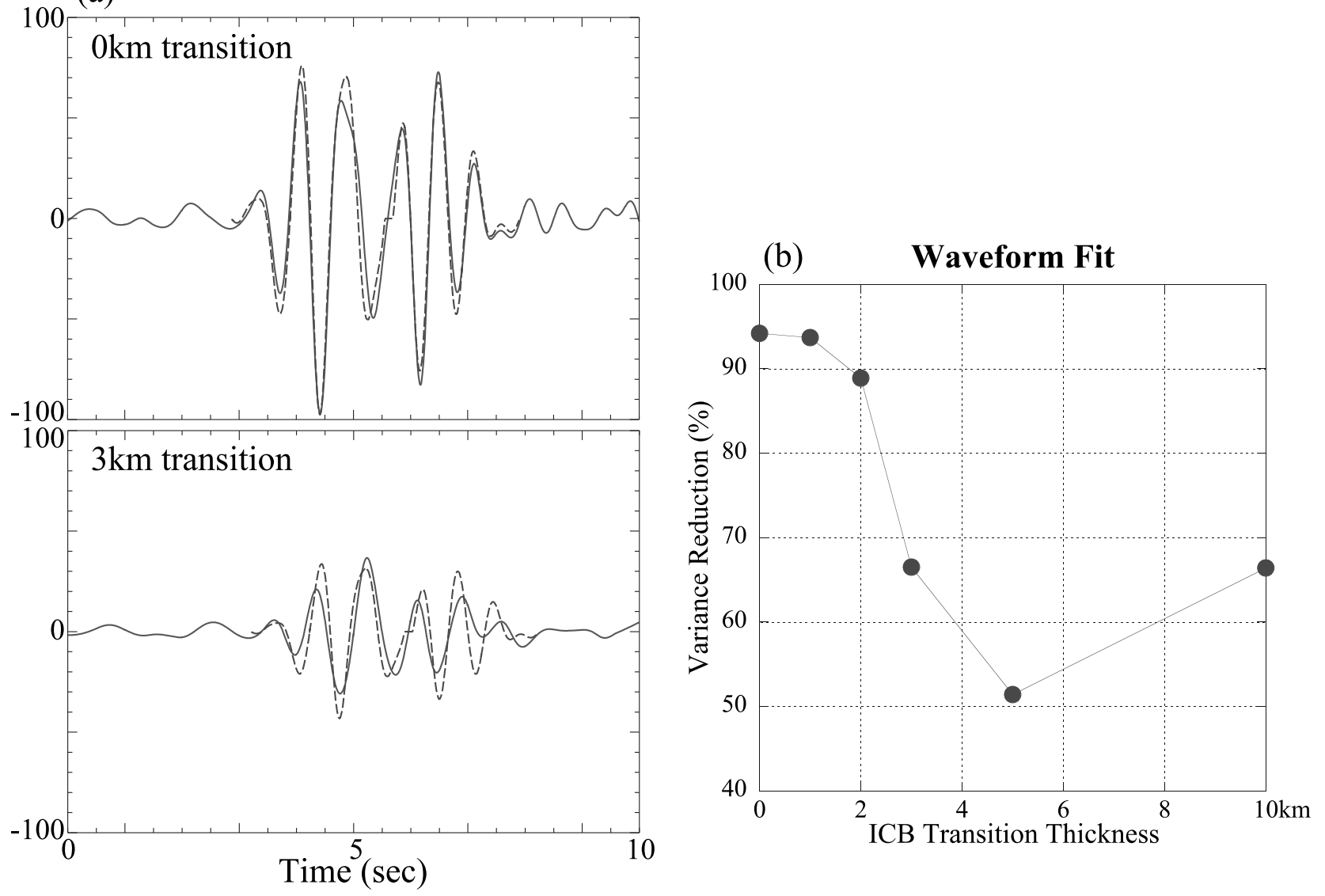

Fig. 6. The ICB transition thickness and PKiKP waveform fit. The observed (stacked) PKiKP is compared with synthetic PKiKPs constructed from the observed (stacked) PcP assuming a finite thick ICB. (a) Waveforms are compared for 0 and 3 km-thick ICB. Solid lines and broken lines are simulated and observed PKiKPs, respectively. The maximum absolute amplitude of the simulated PKiKP for a 0-km thick ICB is set to 100. (b) The variance reduction is plotted as a function of the ICB thickness. A 0-km thick ICB gives the best fit.

reverberation of $\mathrm{pPKiKP}$ ). If so, however, we should observe a similar phase for PcP stacks. Figure 8(b) compares stacks for PKiKP and PcP. In both cases, the surface reflection phases, $\mathrm{pPKiKP}$ and $\mathrm{pPcP}$ are observed at similar times. On the other hand, no second phase appears for the PcP stack, which deserves some consideration.

One explanation may be to attribute it to a near-surface structure (ocean and crust). Although the surface reflection points of observed $\mathrm{pPKiKP}$ and $\mathrm{pPcP}$ are closely distributed, there is some difference if we actually plot them. The surface reflection points of observed pPKiKP are not separated more than $8 \mathrm{~km}$ and appear to be a single point on a map, while those of PcP are separated by as much as 30 $\mathrm{km}$. As these points are located within the back arc region of the Mariana subduction zone, where the crustal thickness and water depth both vary significantly laterally, the noted difference of the distribution of the surface reflection points may affect reflected waveforms. The $10 \mathrm{~s}$ delayed second phase can be caused by a positive interference of water reverberation and crustal reverberation phases; PREM synthetics bandpass filtered between $0.5-1.0 \mathrm{~Hz}$ actually shows a similar $10 \mathrm{~s}$ delayed second phase, which does not show up in higher-frequency intervals. This suggests subtlety in the interference. PREM happens to have a very peculiar shallow structure: P-wave two-way travel times of the water layer and the upper crust layer are identical (4.14 s), which may help this interference effect. As the average depth of the ocean in the area is about $3 \mathrm{~km}$, a similar situation may be actually occurring for the observed pPKiKP. As for $\mathrm{pPcP}$, the spread of the reflection points in this heterogeneous area may disturb the positive interference discussed above. Without the absence of a detailed structure known for the area (H. Takahashi, personal communication, 2003), it is difficult to test this quantitatively.

The alternative explanation is to give the pPKiKP second phase an inner core origin. From the time difference between PKiKP and this phase ( $85 \mathrm{~s}$ ), it can be explained as a reflected phase from a discontinuity $470 \mathrm{~km}$ beneath the ICB. As the slowness of the phase is slightly different from the predicted value for a horizontal discontinuity ( $\Delta p \sim 0.16 \mathrm{~s} / \mathrm{deg}$ ), the discontinuity must be slightly dipping $\left(\sim 5^{\circ}\right)$. From the amplitude, the reflection of this postulated discontinuity is about $3 \%$. It is difficult to discriminate these two possibilities based on the presently available data alone. Records from a similar event of a different depth in the future should help to resolve this. Considering that PREM synthetics actually show a similar behavior for the second phase, at the present moment, we prefer the first explanation.

There are also a few other relatively large amplitude phases which have expected slownesses of reflections from horizontal boundaries beneath the ICB: they are observed at 
(a)
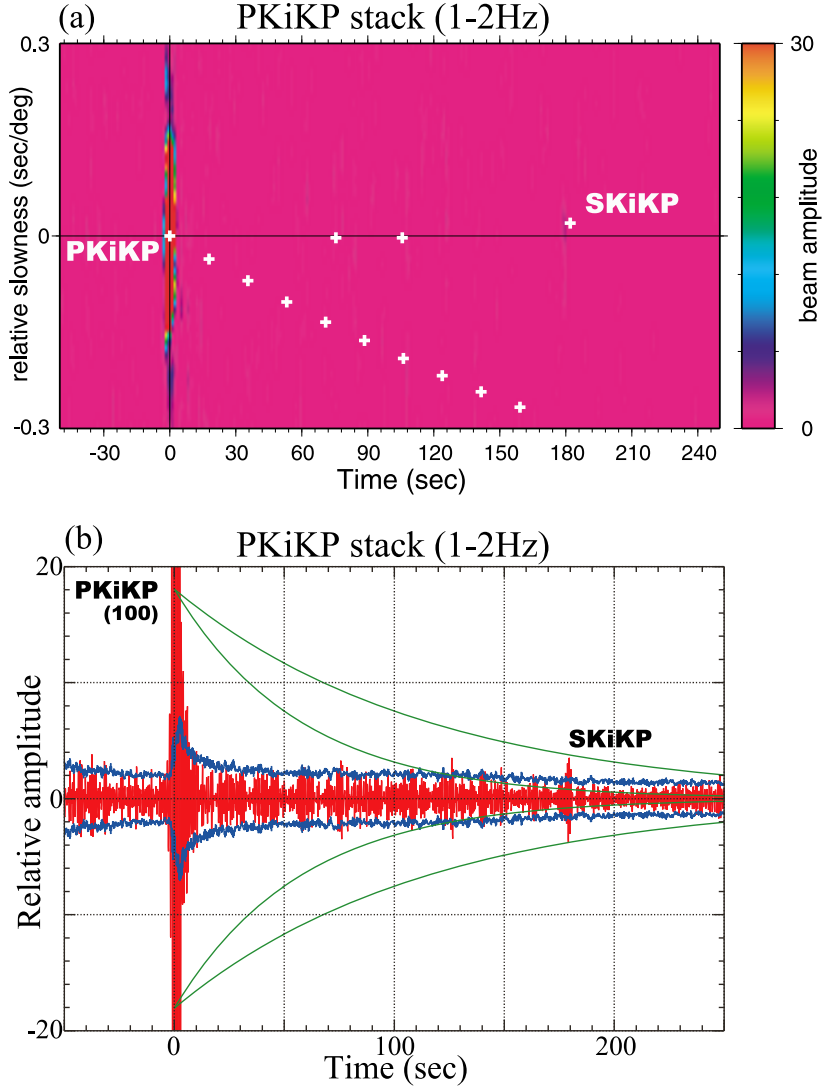

Fig. 7. Stacking performed using PKiKP as a reference phase $(1-2 \mathrm{~Hz})$. (a) Phase-weighted vespagram with the multiplication parameter $v=2$ (Schimmel and Paulssen, 1997). White plus marks denote locations of the ICB related phases (near zero relative slowness) and reflections from horizontal discontinuities below the ICB for every $100 \mathrm{~km}$ interval (negative slowness). (b) Linear stack waveform for the PKiKP slowness. Green lines denote amplitude of $1 \%$ reflection phase at 1 and $2 \mathrm{~Hz}$, and blue lines show two standard deviations. The amplitude of PKiKP is set to 100 .

$\sim 17$, $\sim 30$, and $\sim 41$ s after PKiKP (Fig. 8(a)). The corresponding approximate depth (reflection) of these phases are $100 \mathrm{~km}(1.6 \%), 170 \mathrm{~km}(1.7 \%)$, and $230 \mathrm{~km}(1.5 \%)$, respectively. Although the amplitudes of these phases are well above the two standard deviation level, we try not to overinterpret them, and just nominate them as possible candidates for inner core reflectors/discontinuities which may correspond to the bottom of the low porosity "crust" suggested by Sumita et al. (1996) and Sumita and Yoshida (2003).

From these observations, we may safely conclude that there is no sharp ( $\sim 5 \mathrm{~km}$ thick) reflector/discontinuity in the top $400 \mathrm{~km}$ part of the inner core $(\sim 70 \mathrm{~s}$ after PKiKP) whose reflection amplitude is larger than $\sim 2 \%$ (2\% contrast both in velocity and density if they scale together).

\section{Discussion}

\subsection{The ICB structure}

Sharpness The sharpness of the ICB has been discussed mostly in terms of the frequency content of the observed near-vertical PKiKP. Using LASA array data, Engdahl et al. (1970) observed short-period ( $\sim 1 \mathrm{~Hz})$ PKiKP. This observation was later used by Phinney (1970) and Cummins and Johnson (1988a) to constrain the ICB transition thickness to be about $\sim 1.5 \mathrm{~km}$ and less than $5 \mathrm{~km}$, respectively.

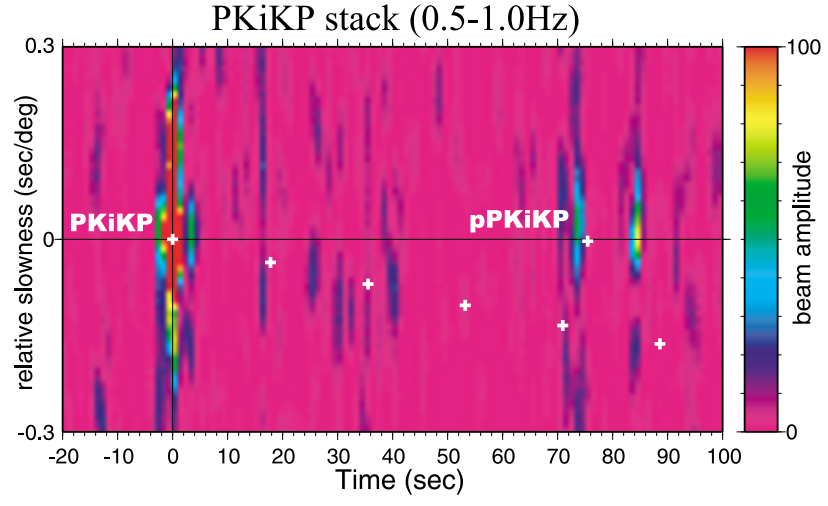

(b)

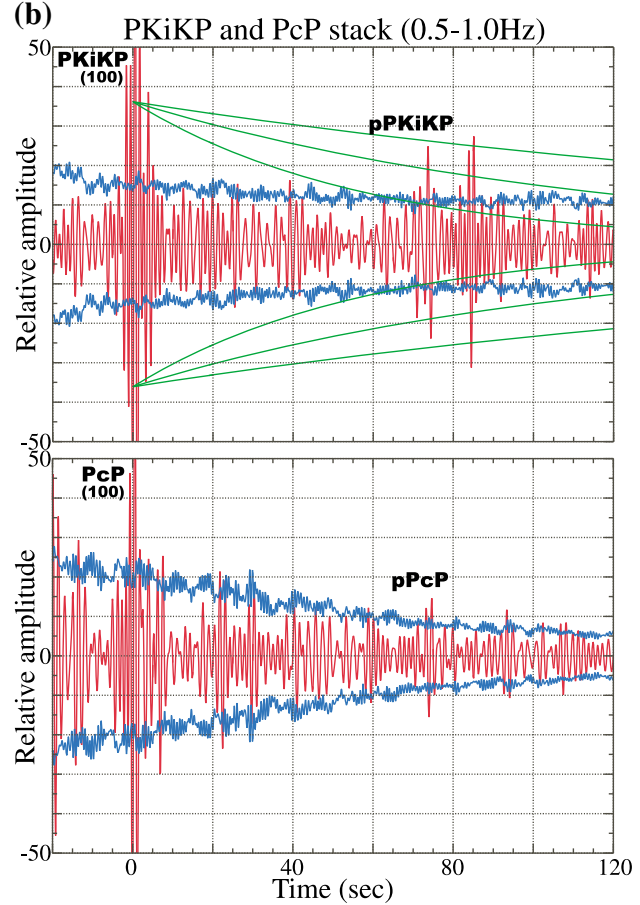

Fig. 8. Stacking performed using PKiKP as a reference phase $(0.5-1 \mathrm{~Hz})$. (a) Similar to Fig. 7(a). (b) Similar to Fig. 7(b), but for both PKiKP and PcP. Here the green lines denote amplitude of $2 \%$ reflection phase at $0.5,1.0$ and $2.0 \mathrm{~Hz}$. Note that a lack of the second phase for $\mathrm{pPcP}$.

The latter researchers noted that the inference of the former one is based on a simplified approach and that they take a more conservative estimate. Our estimate for the ICB transition thickness is based on both amplitude and waveform analyses. We estimate the thickness $0-1 \mathrm{~km}$ (at most $2 \mathrm{~km}$ ), and thus the ICB is a very sharp boundary. As the amplitude ratio of $\mathrm{PKiKP} / \mathrm{PcP}$, which will be discussed in some detail below, is mostly consistent with PREM which has a $0 \mathrm{~km}$ thick boundary, the inner core just below the ICB may have substantial solid material not so different from the deeper part of the inner core. This is consistent with the theoretical consideration by Sumita et al. (1996). They show that the efficient sedimentation compaction expels melt from the inner core and estimate the "mushy zone" to be $\sim 10 \mathrm{~m}$ thick.

PKiKP/PcP Amplitude ratio The amplitude ratio of $\mathrm{PKiKP} / \mathrm{PcP}$ has often been used to constrain the density jump at the ICB (e.g., Souriau and Souriau, 1989). Our estimate of the ratio is $\sim 5 \%$ larger than that of PREM 


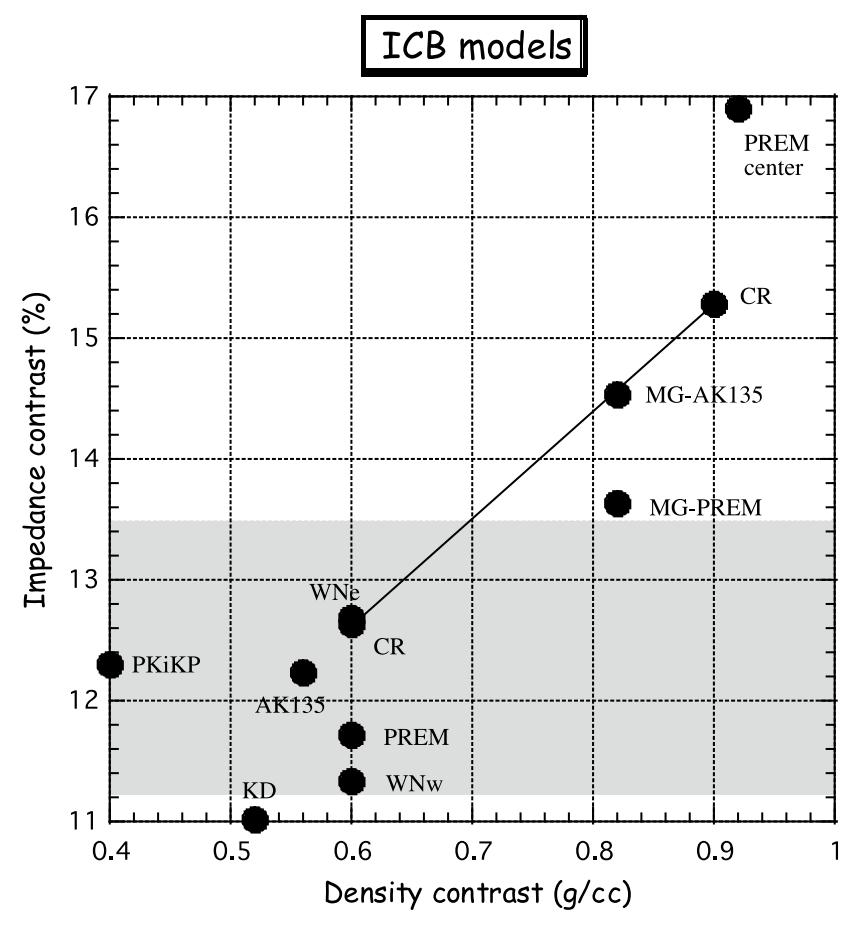

Fig. 9. Models for the ICB given as functions of the impedance and the density contrasts. Models are PREM (Dziewonski and Anderson, 1981); AK135 (Kennett et al., 1995); KD (Koper and Dombrovskaya, 2005); $\mathrm{WN}[\mathrm{w}, \mathrm{e}]$ (western and eastern hemisphere of Wen and Niu, 2002, assuming the PREM density contrast).; MG-[PREM, AK135] (Masters and Gubbins, 2003, assuming PREM and AK135 P-wave velocity contrast, respectively); CR (Cao and Romanowicz, 2004); PREM center (values just below the ICB are taken from those of the center of the Earth of PREM, representative of the possible maximum). Shaded area gives our estimate of the impedance contrast (based on the observation at $\Delta \sim 15^{\circ}$ ).

$\left(14^{\circ}<\Delta<16^{\circ}\right)$, and the estimated error is about $10 \%$, corresponding to two standard deviations. Although this observation alone cannot constrain much of the physical conditions at the top of the inner core, once we know the general feature of the ICB region, our observation may provide some new insight. For example, if PREM represents the real features of the ICB region, our observation requires that the structure of the inner core just below the ICB must have substantial solid material not so different from the deeper part of the inner core.

On the other hand, if a larger density jump at the ICB is preferred (e.g., Masters and Gubbins, 2003; Cao and Romanowizc, 2004), there must be a large gradient in both Pand S-velocities and density structures just below the ICB. Figure 9 summarizes recent published models of the ICB and our observation. It may be safe to conclude that the upper bound for the impedance contrast at the ICB is $\sim 15 \%$. In this case, our observation indicates that the amplitude of PKiKP at $1-2 \mathrm{~Hz}$ is reduced to $20-30 \%$. Assuming that the melt fraction variation is the key controlling factor for the velocity change inside of the inner core, Singh et al. (2000) suggested that the presence of a volume fraction of 3 to $10 \%$ explained the seismic observation. With their preferred model, $\mathrm{P}$ and $\mathrm{S}$ velocities are respectively reduced, $\sim 0.04$ and $\sim 0.125 \mathrm{~km} / \mathrm{s}$ for a $1 \%$ increase of the melt fraction. To make a $10 \%$ reduction of the impedance contrast at the ICB, up to about a $3 \%$ increase in melt fraction (translates into $0.37 \mathrm{~km} / \mathrm{s} \mathrm{S}$-wave velocity reduction) may be required. To account for a $20-30 \%$ reduction, the S-wave velocity just below the ICB may be as low as $\sim 2.5 \mathrm{~km} / \mathrm{s}$, which is in a similar range as some of recent models (Koper and Pyle, 2004; Cao and Romanowicz, 2004). Thus, it appears possible to discriminate different views of the ICB once we know the overall feature of the ICB region.

Amplitude variation Both $\mathrm{PcP}$ and PKiKP show large variations (about a factor of 5) in the observed amplitude. As their statistical properties do not seem to be significantly different and as there is no correlation between the two amplitude observations, we tentatively attribute this variation to the presence of small-scale heterogeneities in the crust and mantle within the ray paths of corresponding phases. As the observed $\mathrm{PKiKP} / \mathrm{PcP}$ ratio also varies by about a similar factor, we need to be cautious to interpret amplitudes in the observed frequency range based on a single or few stations measurement as suggested by Shearer and Masters (1990). On the other hand, it should also be noted that Krasnoshchekov et al. (2005) recently interpreted the variability of the PKiKP amplitude observed for nuclear explosions as evidence for the regional variability of the reflection property of the ICB ("mosaic structure"). In this view, the studied region of the ICB of the present paper may correspond to a sharp "patch".

Inner core scattering Vidale and Earle (2000) reported a scattered energy timing after the expected arrivals of PKiKP around $\Delta \sim 70^{\circ}$ where PKiKP has a small expected amplitude in the global reference models (PREM, AK135), and attributed it to the presence of scatterers in the top $300 \mathrm{~km}$ part of the inner core. We have attempted to observe such signals (gradual grow-up of background amplitudes) after our PKiKP arrivals by various stacking techniques (e.g., reducing array size, envelope stacking, etc.), but are unable to observe any signature. A simplified estimate of the scattered energy (a vertical plane P-wave incidence observed $5000 \mathrm{~km}$ away) based on Wu and Aki's (1985) theory predict the amplitude of the scattered energy at about several percent of the input PKiKP for Vidale and Earle's model. As this is hard to observe in our data, their model does not seem to be inconsistent with our data. On the other hand, the model suggested by Cormier and Li (2002) predicts much larger scattered energy (similar to the input PKiKP amplitude) that should be observed in the present data. As their model is the maximum one for attributing all the cause of the attenuation to the scatterers, absence of such a large scattered energy indicates that there exists substantial intrinsic attenuation in the inner core.

\subsection{Inner core discontinuity?}

There have been suggestions of the presence of discontinuous transition zones in the inner core. Souriau and Souriau (1989) indicated an observation of a possible sharp (observable around $1 \mathrm{~Hz}$ ) discontinuity about $120 \mathrm{~km}$ below the ICB in the region now classified as the "eastern hemisphere" (Tanaka and Hamaguchi, 1997; Niu and Wen, 2001). As they presented no slowness analysis, it is hard 
to access the reliability of their observation. Song and Helmberger (1998) also reported 4\% P-wave velocity jump at a depth of $95 \mathrm{~km}$ in the "western hemisphere". Recently, Leyton et al. (2005) combined many PKiKP observations of Koper et al. (2003) to infer the discontinuity structure within the inner core, and concluded the absence of strong ( $>3 \%$ reflection) global sharp discontinuity in the inner core. As to more transitional change $(<50 \mathrm{~km})$, Song and Helmberger (1998) reported a large velocity jump of $4.3 \%$ at a depth of $250 \mathrm{~km}$ in the "western hemisphere", and suggested that it corresponded to the boundary between the shallow isotropic layer and the anisotropic deeper part. Also, Stroujkova and Cormier (2004) indicated a sharp velocity increase $(\sim 3 \%)$ at a depth of $40 \mathrm{~km}$ in the equatorial region of the "eastern hemisphere".

Our observation, by far presents the best constraint for the presence of sharp discontinuities. Nevertheless, as noted earlier, we could not find strong evidence for such a discontinuity. The strongest possible discontinuity is observed for a depth of $470 \mathrm{~km}$ below the ICB. As our observation region is within the "eastern hemisphere" of the inner core where a thicker $(\sim 400 \mathrm{~km})$ isotropic surface layer is reported (e.g., Ouzounis and Creager, 2001; Wen and Niu, 2002; Souriau et al., 2003), the observed signal may correspond to the bottom of this layer. We, however, note again that this signal may be related to the crustal reverberation of pPKiKP. Besides this observation, our records indicate that there is no sharp discontinuity within the top $400 \mathrm{~km}$ of the inner core with a reflection amplitude of $2 \%$, although a few candidates exist for discontinuities with smaller reflections $(\sim 1.5 \%)$.

We have also attempted to observe a reflected phase from the other side of the ICB, PKIIKP, but not succeeded in observing any. Bolt (1977) reported a LASA observation of the amplitude ratio PKIIKP/PKiKP to be $\sim 4 \%$. Considering the noise level, the lack of PKIIKP signals in our date is consistent with Bolt's observation which constrains the average $Q$ of the inner core $\bar{Q}=450 \pm 100$.

\section{Conclusion}

We have presented an unusual entire network observation of near-vertical PKiKP which gives constraints on the structure at and below the inner core boundary. Our results indicate that the ICB is a very sharp boundary $(0-1 \mathrm{~km}$ thick; no more than $2 \mathrm{~km}$ thick); this is consistent with the result of Sumita et al. (1996) who predicted a thin mushy zone at the ICB. The structure below the ICB is relatively transparent, with no sharp ( $\sim 5 \mathrm{~km}$ thick) horizontal discontinuity in the top $400 \mathrm{~km}$ part of the inner core whose reflection amplitude is larger than $\sim 2 \%$. We observed one possible reflected phase from a slightly dipping $\left(\sim 5^{\circ}\right)$ reflector/discontinuity inside of the inner core around a depth of $470 \mathrm{~km}$ below the ICB (3\% reflection). However, this phase may be due to $\mathrm{pPKiKP}$ water/crustal layer reverberations. The bounce points of PKiKP at the ICB are located in an area about of $8^{\circ}(\sim 171 \mathrm{~km}$ at the ICB $)$, which is comparable to the size of the Fresnel zone. The estimated properties of the ICB region correspond to an average feature of at least this size.

Acknowledgments. The author thanks Narumi Takahashi for the information regarding the structure in the Mariana back arc region, Nozomu Takeuchi for the DSM synthetics, Yoshio Fukao for discussion on the phase delay, and Yoshiko Yamanaka for the source mechanism information. A part of the research was conducted while the author was a visiting professor at IPGP in 2003. He thanks the members of IPGP, especially to Jean-Paul Montagner for hospitality. Comments by Azusa Shito and Keith Koper improved the manuscript. GMT (Wessel and Smith, 1995) is used to create some of the figures.

\section{References}

Bhattacharyya, J., P. Shearer, and G. Masters, Inner core attenuation from short-period $\mathrm{PKP}(\mathrm{BC})$ versus $\mathrm{PKP}(\mathrm{DF})$, Geophys. J. Int., 114, 1-11, 1993.

Bolt, B., The detection of PKIIKP and damping in the inner core, Ann. Geofis., 30, 507-520, 1977.

Cao, A. and B. Romanowicz, Constraints on density and shear velocity contrasts at the inner core boundary, Geophys. J. Int., 157, 1146-1151, 2004.

Cormier, V. F. and X. Li, Frequency-dependent seismic attenuation in the inner core 2. A scattering and fabric interpretation, J. Geophys. Res., 107(B12), 2362, doi:10.1029/2002JB001796, 2002.

Creager, K. C., Anisotropy of the inner core from differential travel times of the phases PKP and PKIKP, Nature, 356, 309-314, 1992.

Cummins, P. and L. R. Johnson, Short-period body wave constraints on properties of the Earth's inner core boundary, J. Geophys. Res., 93, 9058-9074, 1988

Dziewonski, A. M. and D. L. Anderson, Preliminary reference Earth model, Phys. Earth Planet. Inter., 25, 297-356, 1981.

Engdahl, E. R., E. A. Flinn, and C. F. Romney, Seismic waves reflected from Earth's inner core, Nature, 228, 852-1053, 1970.

Fearn, D. R., D. E. Loper, and P. H. Roberts, Structure of Earth's inner core, Nature, 292, 232-233, 1981.

Kawakatsu, H. and S. Watada, Japanese seismic array as a probe for Earth's deep interior, Programme and Abstracts, Seism. Soc. Japan, A46, 2002.

Kennett, B., E. Engdahl, and R. Buland, Constraints on seismic velocities in the Earth from traveltimes, Geophys. J. Int., 122, 108-124, 1995.

Koper, K. D. and M. Dombrovskaya, Seismic properties of the inner core boundary from PKiKP/P amplitude ratios, Earth Planet. Sci. Lett., 237, 680-694, 2005.

Koper, K. D. and M. L. Pyle, Observations of PKiKP/PcP amplitude ratios and implications for Earth structure at the boundaries of the liquid core, J. Geophys. Res., 109, B03301, doi:10.1029/2003JB002750, 2004.

Koper, K. D., M. L. Pyle, and J. M. Franks, Constraints on aspherical core structure from PKiKP-PcP differential travel times, J. Geophys. Res., 108(B3), 2168, doi:10.1029/2002JB001995, 2003.

Koper, K. D., J. M. Frank, and M. Dombrovskaya, Evidence for smallscale heterogeneity in Earth's inner core from a global study of PKiKP coda waves, Earth Planet. Sci. Lett., 228, 227-241, 2004.

Krasnoshchekov, D. N., P. B. Kaazik, and V. M. Ovtchinnikov, Seismological evidence for mosaic structure of the surface of the Earth's inner core, Nature, 435, 483-487, 2005.

Labrosse, S. and M. Macouin, The inner core and the geodynamo, $C . R$. Geoscience, 335, 37-50, 2003.

Lehmann, I., P', Bur. Centr. Seism. Internat., A, 14, 3-31, 1936.

Leyton, F., K. D. Koper, L. Zhu, and M. Dombrovskaya, On the lack of seismic discontinuities within the inner core, Geophy. J. Inter., 162, 779-786, 2005.

Masters, G. and D. Gubbins, On the resolution of density within the Earth, Phys. Earth Planet. Int., 140, 159-167, 2003.

Morelli, A., A. M. Dziewonski, and J. H. Woodhouse, Anisotropy of the inner core inferred from PKIKP travel times, Geophys. Res. Lett., 13 1545-1548, 1986

Niu, F. and L. Wen, Hemispherical variations in seismic velocity at the top of the Earth's inner core, Nature, 410, 1081-1084, 2001.

Obara, K., K. Kasahara, S. Hori, and Y. Okada, A densely distributed highsensitivity seismograph network in Japan: Hi-net by National Research Institute for Earth Science and Disaster Prevention, Review Scientific Instruments, 76, 021301, doi:10.1063/1.1854197, 2005.

Obayashi, M. and Y. Fukao, P and PcP travel time tomography for the core-mantle boundary, J. Geophys. Res., 102, 17825-17841, 1997.

Ouzounis, A. and K. Creager, Isotropy overlying anisotropy at the top of the inner core, Geophys. Res. Lett., 28, 4331-4334, 2001.

Phinney, R. A., Reflection of acoustic waves from a continuously varying 
interfacial refion, Rev. Geophys. Space Phys., 8, 517-532, 1970.

Poupinet, G. and B. L. N. Kennett, On the observation of high frequency PKiKP and its coda in Australia, Phys. Earth Planet. Int., 146, 497-511, 2004.

Schimmel, M. and H. Paulssen, Noise reduction and detection of weak, coherent signals through phase-weighted stacks, Geophys. J. Int., 130, 497-505, 1997.

Shearer, P. and G. Masters, The density and shear velocity contrast at the inner core boundary, Geophys. J. Int., 102, 198-491, 1990.

Shimizu, H., J. P. Poirier, and J. L. Le Mouel, On crystallization at the inner core boundary, Phys. Earth Planet. Int., 151, 37-51, 2005.

Singh, S. C., M. A. J. Taylor, and J.-P. Montagner, On the presence of liquid in Earth's inner core, Science, 287, 2471-2774, 2000.

Song, X. D. and D. V. Helmberger, Anisotropy of Earth's inner core, Geophys. Res. Lett., 20, 2591-2594, 1993.

Song, X. D. and D. V. Helmberger, Seismic evidence for an inner core transition zone, Science, 282, 924-927, 1998.

Song, X. D. and P. G. Richards, Observational evidence for differential rotation of the Earth's inner core, Nature, 382, 221-224, 1996.

Souriau, A. and M. Souriau, Ellipticity and density at the inner core boundary from subcritical PKiKP and PcP data, Geophys. J. Int., 98, 39-54, 1989.

Souriau, A., R. Garcia, and G. Poupinet, The seismological picture of the inner core: structure and rotation, C. R. Geoscience, 335, 51-63, 2003.

Stroujkova, A. and V. F. Cormier, Regional variations in the uppermost $100 \mathrm{~km}$ of the Earth's inner core, J. Geophys. Res., 109, doi:10.1029/ 2004JB002976, 2004.

Sumita, I. and S. Yoshida, Thermal Interactions between the mantle, outer and inner cores, and the resulting structural evolution of the core, in
Earth's Core: Dynamics, Structure, Rotation, Geodynamics Ser, vol. 31, edited by V. Dehant et al., pp. 213-231, AGU, Washington D.C., 2003.

Sumita, I., S. Yoshida, M. Kumazawa, and Y. Hamano, A model for sedimentary compaction of a viscous medium and its application to innercore growth, Geophys. J. Int., 124, 502-524, 1996.

Takeuchi, N., R. J. Geller, and P. R. Cummins, Highly accurate P-SV complete synthetic seismograms using modified DSM operators, Geophys. Res. Lett., 23, 1175-1178, 1996.

Tanaka, S. and H. Hamaguchi, Degree one heterogeneity and hemispherical variation of anisotropy in the inner core from PKP(BC). PKP(DF) times, J. Geophys. Res., 102, 2925-2938, 1997.

Thorne, M. S. and E. J. Garnero, Inferences on ultralow-velocity zone structure from a global analysis of SPdKS waves, J. Geophys. Res., 109, B08301, doi:10.1029/2004JB003010, 2004.

Vidale, J. E. and P. S. Earle, Fine-scale heterogeneity in the Earth's inner core, Nature, 404, 273-275, 2000.

Wen, L. and F. Niu, Seismic velocity and attenuation structures in the top of the Earth's inner core, J. Geophys. Res., 107(B11), 2273, doi:10.1029/ 2001JB000170, 2002.

Wessel, P. and W. H. F. Smith, New version of the Generic Mapping Tools released, Eos Trans. AGU, 76, 329, 1995.

$\mathrm{Wu}, \mathrm{R}$. S. and K. Aki, Elastic wave scattering by a random medium and the small-scale inhomogeneities in the lithosphere, J. Geophys. Res., 90, 10261-10273, 1985.

H. Kawakatsu (e-mail: hitosi@eri.u-tokyo.ac.jp) 